\title{
Rendimiento cuántico de producción de radical hidroxilo por medidas de fluorescencia en el sistema TCPPFe/ $\mathrm{H}_{2} \mathrm{O}_{2} /$ UV-VIS
}

\author{
The Hydroxyl Radical $\left(\Phi_{\bigcirc \mathrm{H}}\right)$ Production's Quantic Yield, by \\ Fluorescence in the TCPPFe/ $\mathrm{H}_{2} \mathrm{O}_{2} / \mathrm{NV}$-VIS Sustem
}

Fecha de Recepción: 04 de Enero de 2014

Fecha de Aprobación: 02 de Febrero de 2014
Carlos Enrique Díaz-Uribe* William Andrés Vallejo-Lozada* Lizeth Johanna Alvarado-Rueda***

\section{Resumen}

El rendimiento cuántico de producción de radical hidroxilo $\left(\Phi_{\mathrm{OH}}\right)$ fue medido desde la fotólisis del peróxido de hidrógeno $\left(\mathrm{H}_{2} \mathrm{O}_{2}\right)$ catalizada por la tetracarboxifenilporfirina de hierro (III) (TCPPFe) bajo luz ultravioleta. $\Phi_{\mathrm{OH}}$ fue determinado por medidas de fluorescencia delproducto de reacción entre el radical hidroxilo $\left(\mathrm{HO}^{\bullet}\right)$ y el tereftalato disódico. La TCPPFe presenta un $\Phi_{\mathrm{OH}}=0,13$, mientras que la reacción estándar de la fotólisis del $\mathrm{H}_{2} \mathrm{O}_{2}$, no catalizada, tiene un $\Phi_{\mathrm{OH}}=0,11$. La reacción no tiene lugar en ausencia de luz, pero en presencia de luz visible, $\mathrm{H}_{2} \mathrm{O}_{2}$ y TcPPFe generan $\mathrm{HO}^{\bullet}$.

Palabras clave: Radical hidroxilo; Porfirina; Rendimiento cuántico.

\section{Abstract}

The quantum yield to production of hydroxyl radical $\left(\Phi_{\mathrm{OH}}\right)$ was measured from the photolysis of hydrogen peroxide $\left(\mathrm{H}_{2} \mathrm{O}_{2}\right)$ catalyzed by tetracarboxy-phenyl-porphyrin $\mathrm{Fe}$ (III) (TCPPFe) under UV irradiation. $\Phi_{\mathrm{OH}}$ was determined by fluorescence measurements by the reaction product between hydroxyl radical $\left(\mathrm{HO}^{\bullet}\right)$ and the disodium terephthalate. The TCPPFepresented a $\Phi_{\mathrm{OH}}=0.13$ while the standard uncatalyzed photolysis reaction of $\mathrm{H}_{2} \mathrm{O}_{2}$ presented a $\Phi_{\mathrm{OH}}=0.11$. Under light irradiation on $\mathrm{H}_{2} \mathrm{O}_{2}$ and TcPPFe, the radical $\mathrm{HO}^{\bullet}$ was generated and, the reaction did not occur in the absence of light.

Keywords:Hydroxyl radical,Porphyrin, Quantum yield.

\footnotetext{
* Ph.D.Universidad del Atlántico (Barranquilla, Atlántico - Colombia). carlosdiaz@mail.uniatlantico.edu.co

** Ph.D. Universidad del Atlántico (Barranquilla, Atlántico - Colombia ). williamvallejo@mail.uniatlantico.edu.co

*** M.Sc. Universidad Santo Tomás (Bucaramanga, Santander - Colombia). lizethjohannnaalvaradorueda@mail.ustabuca.edu.co
} 


\section{INTRODUCCIÓN}

La detección y cuantificación de las especies reactivas del oxígeno recibe hoy en día gran interés debido a su relación en procesos químicos, biológicos y ambientales [1-4]. En muy corto tiempo de vida de los radicales requiere para su detección métodos más sensibles y elaborados. La técnica de resonancia paramagnética electrónica (EPR) es la única técnica que específicamente detecta en forma directa espines no apareados; sin embargo, esta técnica presenta algunas limitaciones, como la baja sensibilidad en medios acuosos y por las condiciones de temperatura [5]. La técnica defluorescencia es unabuena alternativa para la identificación del radical hidroxilo, debido a su alta sensibilidad, a su respuesta lineal a un rango de concentraciones de especies reactivas del oxígeno y a su bajo background fluorescente [6]. El radical hidroxilo $\left(\mathrm{HO}^{\bullet}\right)$ puede ser identificado debido a la fluorescencia del 2-hidroxitereftalato disódico (Fig.1), que es formado entre el $\mathrm{HO}^{\bullet}$ y el tereftalato disódico. El 2-hidroxitereftalato disódico presenta una señal con un máximo en $425 \mathrm{~nm}$ [7].

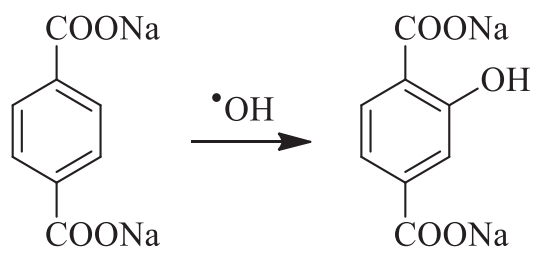

Fig. 1. Reacción del radical hidroxilo y el tereftalatodisódico

Entre las maneras de generar radicales hidroxilo se encuentran las reacciones tipo Fenton y fotoFenton, que utilizan para tal fin peróxido de hidrógeno, sales ferrosas y luz UV [8,9]. Bajo irradiación de la luz UV,el $\mathrm{Fe}^{3+}$ es continuamente reducido a $\mathrm{Fe}^{2+}$, y la reacción Fenton comienza nuevamente; además, las concentraciones son de órdenes de magnitud menores que en la reacción Fenton convencional[10].

En el presente trabajo se aplicó la técnica de fluorescencia para la detección y cuantificación del $\mathrm{HO}^{\bullet}$ formado en la fotólisis del peróxido de hidrógeno $\left(\mathrm{H}_{2} \mathrm{O}_{2}\right)$ catalizada por la tetracarboxifenilporfirina de hierro (III) (TCPPFe) bajo luz UV y visible. Se encontró que la TCPPFe tiene un rendimiento cuántico de producción de radical hidroxilo de 0,2 en presencia de luz UV, $y$, además, que en presencia de luz visible $(\lambda>$ $500 \mathrm{~nm}$ ) se genera radical hidroxilo; esto puede indicar que hay una transferencia electrónica entre la tetracarboxifenilporfirina y el metal.

\section{Materiales y MÉtodos}

\section{A. Síntesis y caracterización del fotocatalizador}

La tetracarboxifenilporfirina sin metal fue sintetizada de acuerdo con el siguiente método: El pirrol, previamente destilado (0.08 moles), fue adicionado a una mezcla de 4-carboxybenzaldehído (0.08 moles), ácido propiónico $(150 \mathrm{~mL})$ y nitrobenceno $(45 \mathrm{~mL})$; posteriormente fue calentado durante una hora a $120{ }^{\circ} \mathrm{C}$; después de enfriarse y removerse el solvente al vacío, la porfirina fue disuelta en 250 $\mathrm{mL}$ de $\mathrm{NaOH} 0.1 \mathrm{M}$. La porfirina fue precipitada con una solución de $\mathrm{HCl}$ 0.1 M. El sólido obtenido se lavó con metanol [11].

La porfirina de hierro fue preparada colocando a reflujo la TCPP $(0.33 \mathrm{mmol})$ con cloruro de hierro hexahidratado $(1.82 \mathrm{mmol})$ en $70 \mathrm{~mL}$ de dimetilformamida por 2 horas y en ambiente de nitrógeno. El DMF fue destilado y la porfirina precipitada al adicionar agua. El sólido obtenido se lavó con $\mathrm{NaOH}$ y $\mathrm{HCl}$. La cantidad de $\mathrm{Fe}$ es 0,025 moles por mg de TCPPFe. FT-IR $\left(\mathrm{KBr}, \mathrm{cm}^{-}\right.$ $\left.{ }^{1}\right)$ : $[1605 \vee(\mathrm{C}=\mathrm{C})]$; $[3411 \vee(\mathrm{O}-\mathrm{H})]$; [1689 $\vee(\mathrm{C}-$ $\mathrm{N})$ ]; $[1268 \vee(\mathrm{C}-\mathrm{O})]$; [1699 $\vee(\mathrm{C}=\mathrm{O})]$.

Los espectros UV-Vis fueron tomados en un espectrofotómetro UV-Vis HP 8453. Los espectros infrarrojos se tomaron en un espectrofotómetro marca Bruker modelo Tensor 27, la cuantificación de hierro presente en la TcPPFe se realizó en un espectrofotómetro de absorción atómica PerkinElmer modelo 372. 


\section{B. Generación y detección del radical hidroxilo}

Para la generación de radical hidroxilo se utilizaron $0,12 \mathrm{mmol}$ de TCPPFe en $10 \mathrm{~mL}$ de una solución acuosa de tereftalato disódico $(4 \mathrm{mM})$ a una temperatura de $298 \mathrm{~K}$, y posterior adición de $100 \mu \mathrm{L}$ peróxido de hidrógeno $(30 \%)$ a un $\mathrm{pH}=3$. Para las reacciones en presencia de irradiación ultravioleta se utilizó una lámpara de vapor de mercurio de baja presión $(254 \mathrm{~nm})$, y para radiación visible, una lámpara halógena de $100 \mathrm{~W}$, haciendo pasar la luz por una solución de dicromato de potasio $1 \mathrm{M}$ para remover longitudes de onda $<500 \mathrm{~nm}$. Varias muestras de la solución se colectaron, filtraron y registraron en un espectrofluorímetro SHIMADZU RF-5301PC en modo de emisión a una longitud de onda de excitación de $315 \mathrm{~nm}$. El registro del espectro se hizo entre 350 y $600 \mathrm{~nm}$.

\section{Cálculo del rendimiento cuántico de producción de radical $\mathrm{OH}$}

Para estimar el rendimiento cuántico de la producción de radical $\mathrm{OH}$ durante la iluminación de la TCPPFe se usó la siguiente ecuación [12]:

$$
\Phi_{H O}=\frac{R_{O H}}{I\left(1-10^{-A}\right)}
$$

Donde $\mathrm{R}_{\mathrm{OH}}$ es la velocidad de producción de $\mathrm{HO} \bullet$; I es la cantidad de fotones que entran al sistema por unidad de volumen y por unidad de tiempo (54 $\mu$ Einstens/L.s), y A es la absorbancia inicial de la solución a la longitud de onda de irradiación (UV=254 nm). La concentración del radical hidroxilo fue determinada usando una curva de calibración del tere ftalato disódico hidroxilado; la velocidad de producción se determinó con el valor de la pendiente de la gráfica de ln [HO•] vs.tiempo.

\section{RESUltados Y DiscusióN}

\section{A. Caracterización del fotosensibilizador}

El espectro UV-vis de la TCPP obtenida en este trabajo se muestra en la Fig. 2; en él se observa la banda de absorción Soreta $419 \mathrm{~nm}$ (transición $\left.\mathrm{a}_{\mathrm{lu}}(\pi)-\mathrm{e}_{\mathrm{g}}^{*}(\pi)\right)$, y 4 bandas $\mathrm{Q}(514,548,588$ y 645 $\mathrm{nm})$, características de la porfirina no metálica, correspondientes a transiciones $\mathrm{a}_{2 \mathrm{u}}(\pi)-\mathrm{e}_{\mathrm{g}}^{*}(\pi)[13]$.

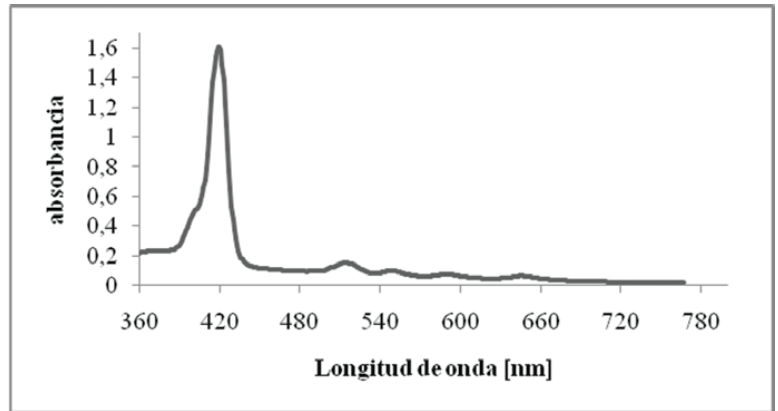

Fig. 2. Espectro UV-vis de la TCPP

El espectro UV-vis de la TCPPFe (Fig.3) exhibe la bandaSoret a 413 nmy 1 banda Q en 534 nm. La disminución del número de bandas $Q$ es típico de las metaloporfirinas. Cuando el ión metálico se coordina con los átomos de nitrógeno del macrociclo, la simetría de la molécula se incrementa y el número de bandas $\mathrm{Q}$ decrece [14]. La metalación de las porfirinas produce desplazamiento de la bandas Soret y Q por la interacción del metal con la nube electrónica $\pi$ de los macrociclos. En el espectro de la TCPPFe se observa un desplazamiento de las bandas de absorción hacia el azul, posiblemente debido a la desestabilización de la nube electrónica $\pi$ del ligando inducida por el metal e incremento de la energía de transición [13].

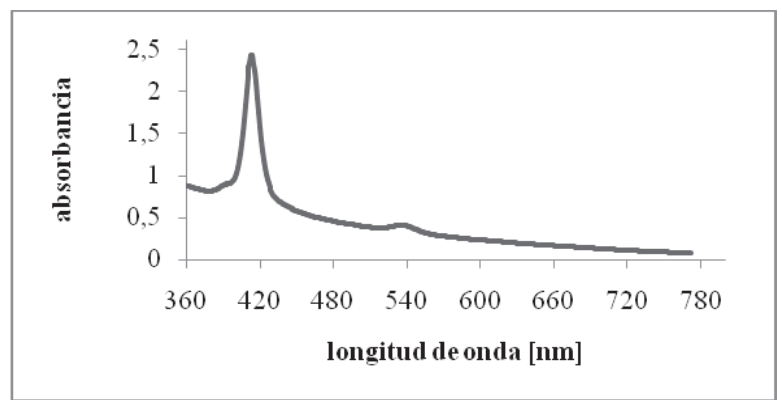

Fig. 3. Espectro UV-vis de la TCPPFe 


\section{B. Generación y detección del radical hidroxilo}

La TcPPFe fue iluminada en presencia de tereftalato disódico y peróxido de hidrógeno con luz UV. El espectro de emisión de fluorescencia (Excitación a $315 \mathrm{~nm}$ ) de la solución fue medido cada 10 minutos durante la irradiación. Como se muestra en la Fig. 4, el incremento gradual en la intensidad de fluorescencia a $425 \mathrm{~nm}$ fue observado con el aumento del tiempo de iluminación.

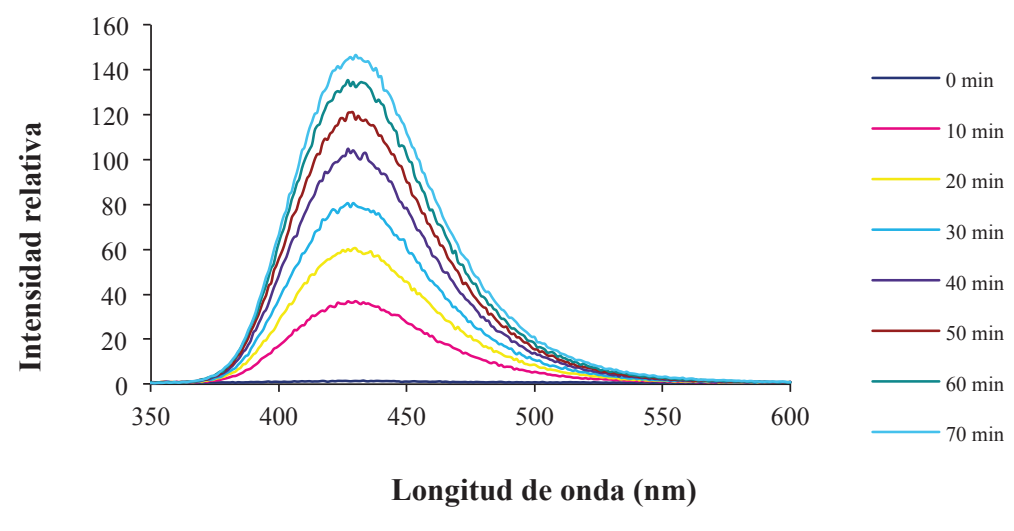

Fig. 4. Espectro de fluorescencia del 2-hidroxitereftalato disódico (excitación $315 \mathrm{~nm}$ )

El espectro generado corresponde al compuesto 2-hidroxitereftalato disódico, producto formado durante la fotocatálisis de la TcPPFe debido a la reacción específica entre el radical hidroxilo y el tereftalatodisódico (Fig. 1).

El radical hidroxilo se genera al usar solo $\mathrm{H}_{2} \mathrm{O}_{2}$ y luz incidente de la lámpara de vapor de mercurio; la irradiación del peróxido de hidrógeno, que involucra longitudes de onda en el ultravioleta, cataliza la producción de radicales hidroxilo, debido a una ruptura del enlace $\mathrm{O}-\mathrm{O}$ del peróxido de hidrógeno. Al adicionar TCPPFe se observó un aumento de la cantidad de radical hidroxilo de $0.25 \mathrm{mM}$ a $0.29 \mathrm{mM}$ (Fig. 5). La interacción de la luz con el hierro (III) lleva a la formación de hierro (II), conduciendo a la generación de radical hidroxilo y regresando nuevamente a hierro (III), continuando el ciclo catalítico.

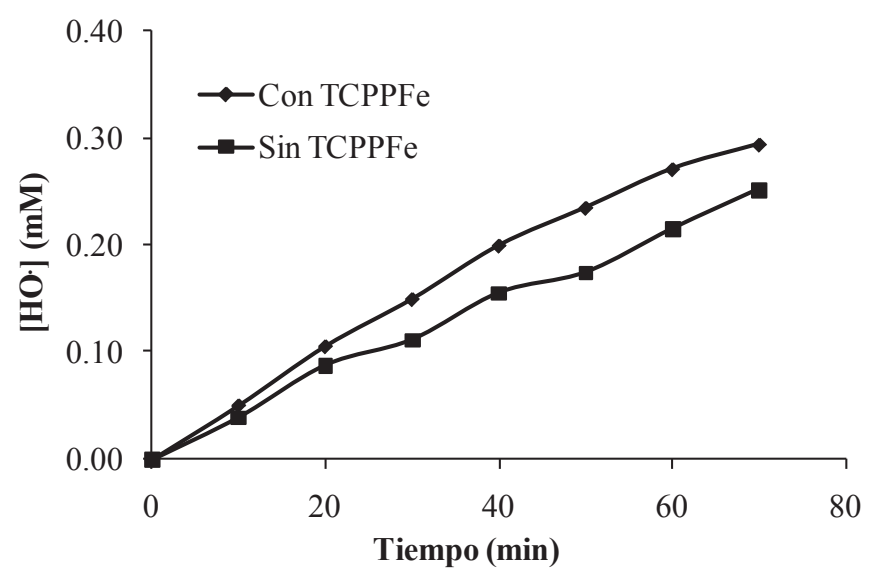

Fig. 5. Formación de 2-hidroxitereftalato disódico en presencia y en ausencia de TCPPFe 
El rendimiento cuántico de producción de radical hidroxilo $\left(\Phi_{\mathrm{HO}}\right)$ se determinó midiendo la fluorescencia del producto de reacción entre el radical hidroxilo y el tereftalato disódico. Se encontró un $\Phi_{\text {но }}$ de 0,13 para la TCPPFe, y uno de 0,11 para la reacción estándar de la fotólisis del $\mathrm{H}_{2} \mathrm{O}_{2}$. La generación de radical $\mathrm{OH}$ no ocurre en ausencia de luz.
Se observó que en presencia de luz visible, en el sistema TCPPFe $/ \mathrm{H}_{2} \mathrm{O}_{2}$ se genera radical hidroxilo (Fig. 6); esto puede indicar que hay una transferencia electrónica entre la tetracarboxifenilporfirina y el metal; por tal motivo, existe un cambio de estado de oxidación de hierro (III) a hierro (II), y la reacción de Fenton ocurre y se produce radical hidroxilo. $\mathrm{La}$ concentración de $\mathrm{HO} \bullet$ estimada en esta reacción fue de $0.06 \mathrm{mM}$.

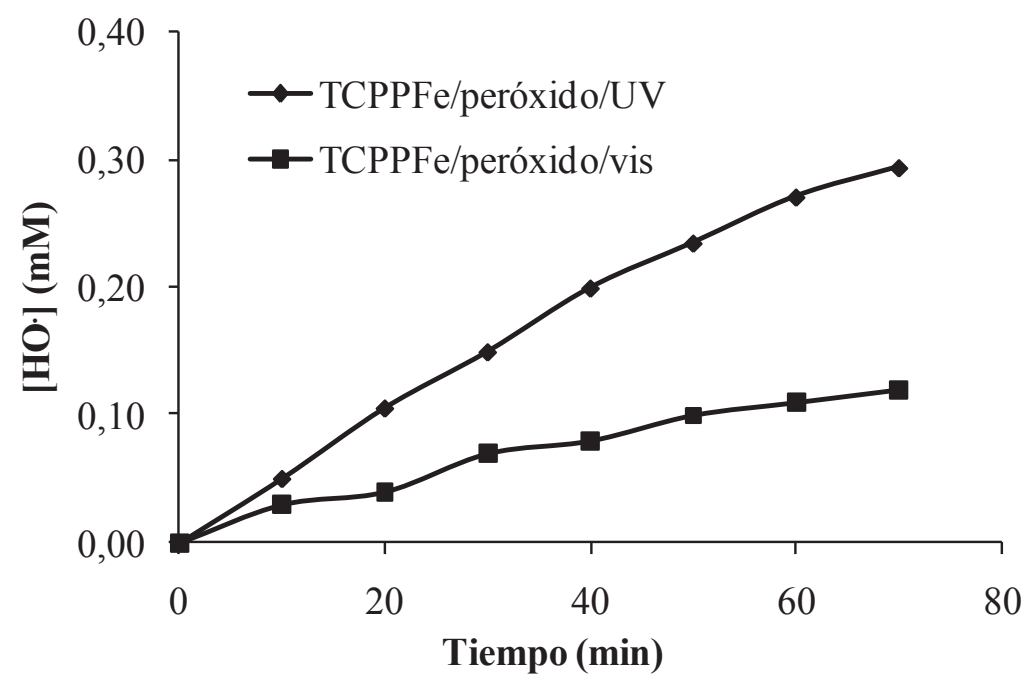

Fig. 6. Formación de 2-hidroxitereftalato disódico en presencia de luz UV y visible

\section{Conclusiones}

En este trabajo se utilizó la sal de tereftalato disódico, para cuantificar la cantidad de radical hidroxilo generado a partir de la reacción de la tetracarboxifenilporfirina de hierro (III) y peróxido de hidrógeno, bajo irradiación UV y visible. Se encontró que la concentración del radical hidroxilo es de $0.29 \mathrm{mM}$ cuando se usa la TcPPFe, y de $0.25 \mathrm{mM}$ en ausencia de éste y en presencia de luz UV. También se obtuvo una actividad del sensibilizador en presencia de luz visible con una cantidad de 0,06 $\mathrm{mM}$ de $\mathrm{HO}$, indicando la transferencia electrónica entre el ligando de la TcPPFe y el metal para el inicio de la reacción de Fenton. Se encontró un $\Phi_{\text {но }}$ de 0,13 para la TCPPFe, y uno de 0,11 para la reacción estándar de la fotólisis del $\mathrm{H}_{2} \mathrm{O}_{2}$.

\section{REFERENCIAS}

[1] W. Freinbichler, L. Bianchi, A. Colivicchi, C. Ballini, K. Tipton, W. Linert and L. Della. "The detection of hydroxyl radicals in vivo", Journal of Inorganic Biochemistry, vol. 102, pp.1329-1333, 2008.

[2] Y. He y D. Hader. "Reactive oxygen species and UV-B: effect on cyanobacteria", Photochemistry and Photobiology Science, vol. 1, pp. 729-736, 2002.

[3] H. Hammel, A. Kapich, K. Jensen and Z. Ryan "Reactive oxygen species as agents of wood decay by fungi", Enzyme and 
Microbial Technology, vol. 30, pp. 445-453, 2002.

[4] P. Wardman. "Fluorescent and luminescent probes for measurement of oxidative and nitrosative species in cells and tissues: Progress, pitfalls, and prospects", Free Radical Biology \& Medicine, vol. 43, pp. 995-1022, 2007.

[5] G. Bartosz. "Use of spectroscopic probes for detection of reactive oxygen species", Clinica Chimica Acta, vol. 368, pp. 53-76, 2006.

[6] K. Ishibashi, A. Fujishima, T. Watanabe and K. Hashimoto "Detection of active oxidative species in $\mathrm{TiO}_{2}$ photocatalysis using the fluorescence technique", Electrochemistry Communications, vol. 2, pp. 207-210, 2002.

[7] K. Ishibashi, A. Fujishima, T. Watanabe and K. Hashimoto "Quantum yields of active oxidative species formed on $\mathrm{TiO}_{2}$ photocatalyst", Journal of Photochemistry and Photobiology A: Chemistry, vol. 134, pp. 134, 139-142, 2000.

[8] M. Tamimi, S. Qourzal, N. Barka, A. Assabbane and Y. Ait-Ichou. "Methomyl degradation in aqueous solutions by Fenton's reagent and the photo-Fenton system", Separation and Purification Technology, vol. 61, pp. 103-108, 2008.
[9] J. Barreiro, M. Duffles and M.Bruun. "Oxidative decomposition of atrazine by a fenton-like reaction in a $\mathrm{H}_{2} \mathrm{O}_{2}$ /ferrihydrite system", Water Research, vol. 41, pp. 5562, 2007.

[10] D. Yingxun, Z. Minghua and L. Lecheng. "Role of the intermediates in the degradation of phenolic compounds by Fenton-like process", Journal of Hazardous Materials, vol. 136,859-865, 2006.

[11] A. Adler, F. Longo, J. Finarelli, J. Goldmacher, J. Assour and L. Korsakoff. "A Simplified Synthesis for MesoTetraphenylporphyrin", Journal of Organic Chemistry, vol. 32, pp. 476, 1967.

[12] E.White, P. P. Vaughan y R. G. Zepp. "Role of the photo-Fenton reaction in the production of hydroxyl radicals and photobleaching of colored dissolved organic matter in a coastal river of the southeastern United States", Aquatic Sciences, vol. 65, pp. 402-414, 2003.

[13] M. Gouterman, G.H. Wagnière and L.C. Snyder. "Spectra of porphyrins: Part II. Four orbital model", Journal of Molecular Spectroscopy, vol. 11, pp. 108-127, 1963.

[14] W. Zheng, N. Shan, L. Yu and Wang X. "UVvisible, fluorescence and EPR properties of porphyrins and metalloporphyrins", Dyes and Pigments, vol 77, pp. 153-157, 2008. 